\title{
Decoupling the Electrical and Entropic Contributions to Energy Transfer from Infrared Radiation to a Power Generator
}

\author{
Aidan L. Gordon, Yosyp Schwab, Brian N. Lang, Graham P. Gearhart, Tara R. Jobin, \\ Justin M. Kaczmar, Zachary J. Marinelli, Harkirat S. Mann, Brian C. Utter*, Giovanna Scarel* \\ Department of Physics and Astronomy, James Madison University, Harrisonburg, USA \\ Email: *utterbc@jmu.edu, scarelgx@jmu.edu
}

Received 19 October 2015; accepted 24 November 2015; published 27 November 2015

Copyright (C) 2015 by authors and Scientific Research Publishing Inc.

This work is licensed under the Creative Commons Attribution International License (CC BY). http://creativecommons.org/licenses/by/4.0/

c) (i) Open Access

\section{Abstract}

The interaction between infrared radiation and a power generator device in time is studied as a route to harvest infrared, and possibly other electromagnetic radiations. Broadening the spectrum of the usable electromagnetic spectrum would greatly contribute to the renewable and sustainable energy sources available to humankind. In particular, low frequency and low power radiation is important for applications on ships, satellites, cars, personal backpacks, and, more generally, where non-dangerous energy is needed at all hours of the day, independent of weather conditions. In this work, we identify an electric and an entropic contribution to the energy transfer from low power infrared radiation to the power generator device, representing electrical and thermal contributions to the power generation. The electric contribution prevails, and is important because it offers multiple ways to increase the voltage produced. For example, placing black-colored gaffer tape on the illuminated face doubles the voltage produced, while the temperature difference, thus the entropic contribution, is not sensitive to the presence of the tape. We recognize the electric contribution through the fast changes it imparts to the voltage output of the power generator device, which mirror the instabilities in time of the infrared radiation. The device thus acts as sensor of the infrared radiation's behavior in time. On the other hand, we distinguish the entropic contribution through the slow changes it causes to the voltage output of the power generator device, which reflect the relative delay with which the two faces of the device respond to thermal perturbations.

\section{Keywords}

Infrared, Power Generators, Energy Harvesting, Electric Contribution

\footnotetext{
${ }^{*}$ Corresponding authors.
}

How to cite this paper: Gordon, A.L., et al. (2015) Decoupling the Electrical and Entropic Contributions to Energy Transfer from Infrared Radiation to a Power Generator. World Journal of Condensed Matter Physics, 5, 301-318. 


\section{Introduction}

A power generator (PG) device can be used to harvest electromagnetic (EM) and, in particular, infrared (IR) radiation. The interaction between the radiation and the device is a complex phenomenon of energy transfer $(\Delta \mathrm{E})$. The rate of energy transferred from the EM radiation per area $a$ of the device is the Poynting vector $|\boldsymbol{S}|=|\boldsymbol{E} \times \boldsymbol{H}|=P / a$, where $\boldsymbol{E}$ and $\boldsymbol{H}$ are the electric and magnetic fields, respectively, and $P$ is power. Therefore, because of $\boldsymbol{E}$ and $\boldsymbol{H}$, the interaction between radiation and device involves the charges on the device surface. Electromagnetic radiation with large frequency $v$ interacts through, e.g., Compton scattering [1], X-ray photoelectron effect [2], photoelectric effect [3], photovoltaic effect [4], and plasmon generation [5]. Electromagnetic radiation with low frequency $v$, e.g. in the IR and microwave regions, resonates with molecular rotation and oscillation frequencies [6] or generates polaritons [7]-[10]. When the photon frequency $v$ or energy $h v$, where $h$ is Planck's constant, do not match with the frequency or the energy of a specific phenomenon involving charges, the energy of the EM radiation contributes to temperature $T$ changes. In photosynthesis this phenomenon is known as internal conversion [11].

We name the energy transferred from the EM radiation to a PG device through the action of the electric $\boldsymbol{E}$ and magnetic $\boldsymbol{H}$ fields as the electric contribution:

$$
\Delta \mathrm{E}_{\mathrm{el}}=q \Delta V,
$$

where $q$ is the charge and $V$ voltage. We name the energy transferred through changes in temperature $T$ at entropy $\Sigma$ as the entropic contribution:

$$
\Delta \mathrm{E}_{\mathrm{en}}=\Sigma \Delta T \text {. }
$$

The energy transferred from IR and microwave radiation is usually associated with the entropic contribution in Equation (2). For example, sun light gives the sensation of temperature increase, and therefore of warmth, on human skin. The microwave radiation in microwave ovens is used to increase the temperature, i.e., cook food and heat-up beverages. Similarly, through laser radiation it is possible to increase temperature, even with nanoscale control [12].

The effects of the electric contribution $\Delta \mathrm{E}_{\mathrm{el}}=q \Delta V$ are less apparent in the energy transfer from low frequency and low power EM radiation. In the current literature, the existence of the electric contribution is acknowledged [13]-[16], but the interplay between the electric and the entropic contributions is not investigated. Specifically, there is a lack of knowledge of 1 ) the possibility of decoupling the electric from the entropic contributions, 2) the factors that promote the electric over the entropic contribution, or vice-versa, 3) the existence of a threshold where one contribution prevails over the other, and 4) the benefits of the electric over the entropic contributions, or vice-versa.

In this work we aim at decoupling $\Delta \mathrm{E}_{\mathrm{el}}$ and $\Delta \mathrm{E}_{\mathrm{en}}$ in a PG device illuminated by low power IR radiation. The device is expected to respond to the entropic contribution by exploiting the Seebeck effect [17]-[20], i.e. producing a voltage difference $\Delta V$ directly proportional to the temperature difference $\Delta T$ applied to the two faces of the PG device, so that $\Delta V=-S \Delta T$. Here, $S$ is the Seebeck coefficient. On the other hand, we expect the PG device to also respond to the electric contribution through its capacitor-type of structure consisting of a sequence of conducting and insulating layers, as illustrated in Figure 1. For the device used in this work, the sequence is, starting from the face illuminated by the IR radiation, a copper $(\mathrm{Cu})$ plate, a layer of pillars made of adoped $\mathrm{Bi}_{2} \mathrm{Te}_{3}$-based alloy, another $\mathrm{Cu}$ plate, and, finally, an alumina (AlO) plate. On the $\mathrm{Cu}$ plates there are electrons whose surface density $\sigma=q / a$ is sensitive to the $\boldsymbol{E}$ and $\boldsymbol{H}$ fields of the IR radiation, thus enabling changes in the electric contribution $\Delta \mathrm{E}_{\mathrm{el}}=q \Delta V$.

In our experiment, the voltage difference $\Delta V(t)$, generated by the PG device through the electric and the entropic contributions, and the temperature difference $\Delta T(t)$, related to the entropic contribution, are observed as a function of time $t$. The measurements capture the first minutes after starting the illumination, and in the 30 hours thereafter. We hypothesize that changes in $|\boldsymbol{S}(t)| \times a=|\boldsymbol{E}(t) \times \boldsymbol{H}(t)| \times a=P(t)$ slowly vary the amplitude of the surface electron density $\sigma(\boldsymbol{r}, t)$. To prove this hypothesis, we study the power $P(t)$ of the IR radiation using a power-meter sensor and compare its behavior with that of $\Delta V(t)$ and $\Delta T(t)$.

Summarizing, we consider the total energy transfer in time from the IR radiation to a PG device as the sum of the electric and the entropic contributions such that:

$$
\Delta \mathrm{E}(t)_{t o t}=q(t) \Delta V(t)+\Sigma \Delta T(t) .
$$




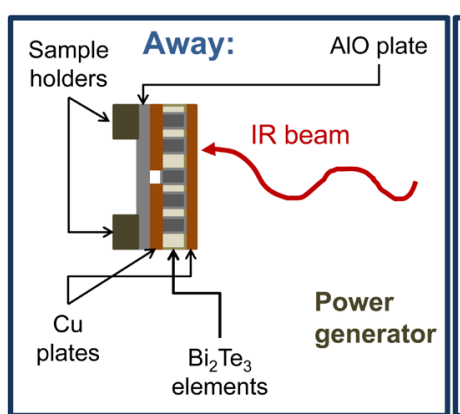

(a)

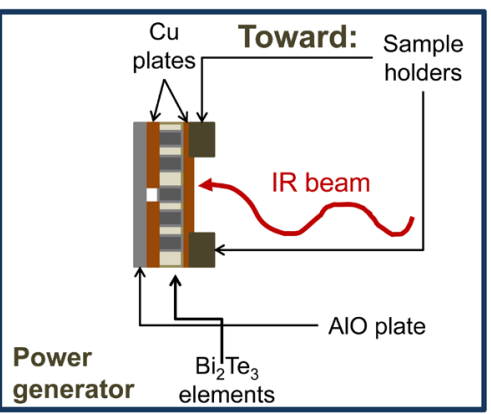

(b)

Figure 1. Schematics of the away (a) and toward (b) architectures of the PG device. In the away architecture (a) the face of the PG device exposed to the IR radiation is free from contact with the sample holder. In the toward architecture (b), the illuminated face is in contact with the sample holder. The PG device is a stack of conducting (Cu plates), non-conducting (AlO plate), and semiconducting (set of pillars made of a doped $\mathrm{Bi}_{2} \mathrm{Te}_{3}$-based alloy) layers.

Consequently, we assume the voltage difference $\Delta V(t)$ produced by the PG device in time to be the addition of two summands:

$$
\Delta V(t)=\frac{\Delta \mathrm{E}(t)_{t o t}}{q(t)}+\left(-\frac{\Sigma \Delta T(t)}{q(t)}\right) .
$$

The first summand relates to the electric and the second to the entropic contribution. The term $S=-\Sigma / q(t)$ can be associated with the Seebeck coefficient.

We will show that with the low power irradiation employed in our measurements, the electric contribution can be decoupled from the entropic contribution, and largely dominates. Decoupling the two contributions is important for IR energy harvesting, because the electric contribution offers a variety of ways to increase the voltage produced by the PG device, e.g. by placing black-colored gaffer tape on the illuminated face of the device, as we will show in Appendix-1. The entropic contribution, instead, is limited by the temperature difference $\Delta T(t)$ established between the two faces of the PG device.

\section{Experimental Set-Up}

For this experiment, continuous broadband IR radiation in the middle IR (MIR) region (i.e. frequency between $350-7500 \mathrm{~cm}^{-1}$, or wavelength between $20-2.2 \mu \mathrm{m}$ ) was produced by a globar (Q301) source. The power $P(t)$ of the IR radiation was monitored versus time using a power-meter sensor Coherent Power Max RS PS19, sensitive to the $300-11000 \mathrm{~nm}$ wavelength range, and to the $100 \mu \mathrm{W}$ to $1 \mathrm{~W}$ power range.

The voltage difference $\Delta V(t)$, generated by the electric and the entropic contributions to $\Delta \mathrm{E}_{\text {tot }}$ according to Equation (4), was produced using a PG device 07111-9L31-04B by Custom Thermoelectric Inc. The device consists of a sequence of layers: 1) a Cu plate on the face exposed to the IR radiation, 2) a layer of pillars made of a doped $\mathrm{Bi}_{2} \mathrm{Te}_{3}$-based alloy, 3) another $\mathrm{Cu}$ plate, and 4) an AlO plate. The Cu plate not illuminated by the IR radiation is non-continuous, as highlighted through the white hole in the left side of Figure 1(a) and Figure 1(b). In the away architecture, illustrated in Figure 1(a), we established the continuity by placing the sample holders, made of anodized aluminum, in contact with the non-continuous $\mathrm{Cu}$ plate. Thus, the $\mathrm{Cu}$ plate together with the sample holder behaves as the electrode of a capacitor. The illuminated Cu plate, instead, was free of contact with the sample holder. In the toward architecture, pictured in Figure 1(b), we left non-continuous the Cu plate opposite to the IR radiation, while the illuminated Cu plate was kept in contact with the sample holder.

The temperatures $T_{I R}(t)$ and $T_{n o-I R}(t)$ of the illuminated and non-illuminated faces, respectively, of the PG device were measured using OMEGA type $\mathrm{E} \mathrm{Ni}-\mathrm{Cr} / \mathrm{Cu}-\mathrm{Ni}$ thermocouple probes. The temperature difference $\Delta T(t)$ was obtained as $\Delta T(t)=T_{I R}(t)-T_{\text {no-IR }}(t)$. The trends of $\Delta V(t), T_{I R}(t)$ and $T_{\text {no-IR }}(t)$ were measured using Keithley 2000 multi-meters. The data were collected using LabView 2012 and a National Instruments PXI-1042q communications chassis.

During the measurements, the PG device and the power-meter sensor were positioned vertically and at an angle of incidence $\theta_{0}=45^{\circ}$ with respect to the IR radiation. The instrumentation was placed in a closed sample 


\section{A. L. Gordon et al.}

compartment purged with $\mathrm{N}_{2}$ to prevent disturbances for the whole duration of the measurements versus time of $P(t), \Delta V(t), T_{I R}(t)$ and $T_{\text {no-IR }}(t)$ [21]. The experimental parameters are summarized in Table 1.

\section{Results and Discussion}

a) Behavior in time of $P(t)$

In the 100 seconds immediately after starting the illumination of the power-meter sensor, $P(t)$, displayed in Figure 2(a), rises exponentially as follows:

Table 1. Summary of the experimental parameters in the main text and in the appendices.

\begin{tabular}{|ccc|}
\hline Main text & & \\
\hline IR frequency $(\mu \mathrm{m})$ & Infrared angle of incidence $\left(^{\circ}\right)$ & PG device final layer \\
$20-2.2$ & 45 & $\mathrm{Cu}$ \\
\hline Appendices & & \\
\hline IR frequency $(\mu \mathrm{m})$ & Infrared angle of incidence $\left(^{\circ}\right)$ & PG device final layer \\
$20-2.2$ & 30 & Alumina \\
\hline
\end{tabular}

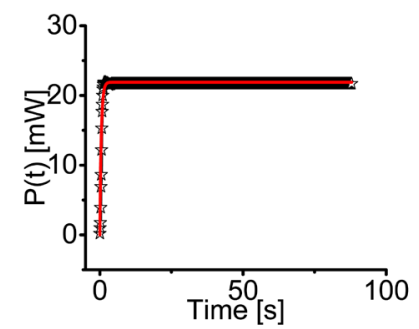

(a)

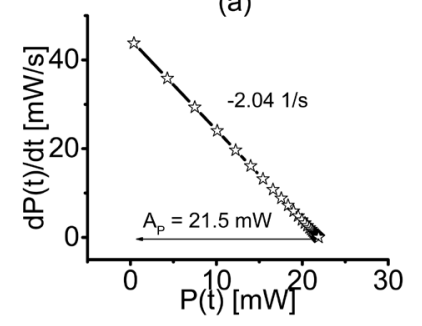

(b)

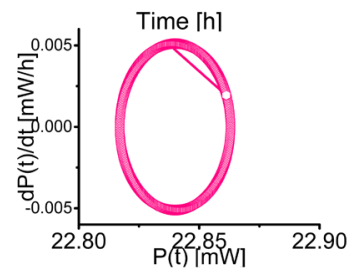

(e)

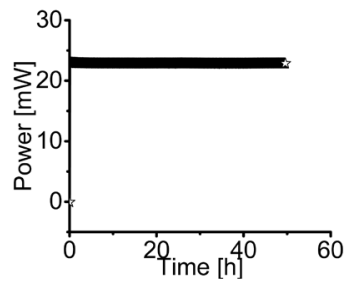

(c)

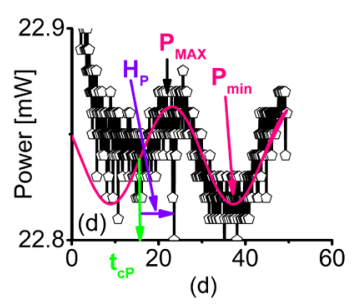

(d)

Figure 2. (a) Exponential rise, as in Equation (5), of the power $P(t)$ versus time of the IR radiation emitted by the globar source in the 100 seconds immediately after starting the illumination of the power-meter sensor; (b) Graph of $\left(\Delta P(t), \frac{\mathrm{d} P(t)}{\mathrm{d} t}\right)$ in the same time interval of (a) reporting the slope and amplitude $A_{P}$; (c) The power $P(t)$ in the 50 hours after starting the illumination of the power-meter sensor; (d) Same as (c), with the vertical scale expanded to highlight the sinusoidal instability region fitted with Equation (6). The zero of the time-scale coincides with the start of the illumination with the IR radiation. The parameters $t_{c P}$ and $H_{P}$ are labeled; (e) Graph of $\left(\Delta P(t), \frac{\mathrm{d} P(t)}{\mathrm{d} t}\right)$ in the 50 hours after starting the illumination of the power-meter sensor. 


$$
P(t)=P_{0} \mathrm{e}^{-\frac{t}{\tau_{P}}}+P_{f},
$$

where $P_{0}$ is the offset, $P_{f}$ the final value, and $\tau_{P}$ the time constant. Typical values of these parameters are reported in Table 2. The slope of the $\left(P(t), \frac{\mathrm{d} P(t)}{\mathrm{d} t}\right)$ graph in Figure 2(b) is negative, indicating the evolution of $P(t)$ toward a stable fixed point [22]. The rate of increase of $P(t)$, i.e. the absolute value of the slope of the $\left(P(t), \frac{\mathrm{d} P(t)}{\mathrm{d} t}\right)$ graph, is $\rho_{P}=2.04 \mathrm{~s}^{-1}$. The amplitude $A_{P}$ of $P(t)$, i.e. the magnitude of the interval along the horizontal scale of the $\left(P(t), \frac{\mathrm{d} P(t)}{\mathrm{d} t}\right)$ graph in Figure 2(b), is $21.5 \mathrm{~mW}$. In the 50 hours after starting the illumination of the power-meter sensor, the power $P(t)$, shown Figure 2(c), reaches a plateau. However, in multiple data sets, we always observe that $P(t)$ undergoes small sinusoidal instabilities shown in Figure 2(d) in which the vertical scale has been expanded. The instability, due to small periodic fluctuations in the closed sample compartment, can be fitted with:

$$
P(t)=P_{\text {off }}+P_{\text {osc }} \sin \left(\frac{t-t_{c P}}{H_{P}}\right),
$$

where $P_{\text {off }}$ and $P_{\text {osc }}$ are the offset value and half of the separation between $P_{\mathrm{MAX}}$ and $P_{\min }$, respectively. The critical time $t_{c P}$ is the point in time in which $P(t)$ reaches $P_{\min }+P_{o s c}$, while $H_{P}$ is the amount of time necessary to move $P(t)$ from $P_{\min }+P_{\text {osc }}$ to $P_{\mathrm{MAX}}$ (practically, 1/4 of the period of the sinusoidal function). The typical values of $P_{o f f}, P_{o s c}, t_{c P}$, and $H_{P}$ are reported in Table 2, labelled in Figure 2(d), and were obtained by placing the zero of the time-scale at the start of the illumination. The instability in $P(t)$ is small, as inferred from $P_{\text {off }} / P_{\text {osc }} \approx 10^{3}$. The $\left(P(t), \frac{\mathrm{d} P(t)}{\mathrm{d} t}\right)$ graph, shown in Figure 2(e), highlights a periodic behavior with frequency $v_{P}=1 / 4 H_{P} \cong 2 \times 10^{-5} \mathrm{~Hz}$ ! We observe the sinusoidal instability of $P(t)$, which modulates the amplitudes of the electric $\boldsymbol{E}$ and magnetic $\boldsymbol{H}$ fields of the IR radiation, to persist beyond the $50 \mathrm{~h}$ time interval in Figure 2(d).

b) Action of the IR radiation on $\sigma(\boldsymbol{r}, t)$

We observed that the power $P(t)$ of the IR radiation rises exponentially obeying Equation (5) at the start of the illumination, and exhibits a sinusoidal instability in the 50 hours thereafter. For the entire time span, we hypothesize that the IR radiation transfers energy, through electric contribution $\Delta \mathrm{E}_{\mathrm{el}}$, to the surface density

Table 2. (Top rows) Fitting parameters $P_{0}, P_{f}$, and $\tau_{P}$ of the IR power $P(t)=P_{0} \mathrm{e}^{-\frac{t}{\tau_{p}}}+P_{f}$ in Equation (5) in the 100 seconds immediately following the start of the illumination of the power-meter sensor with IR radiation from the globar source. The rate of increase of the power $\left(\rho_{P}\right)$, and the amplitude $A_{P}$ in this time interval, derived from the $\left(P(t), \frac{\mathrm{d} P(t)}{\mathrm{d} t}\right)$ graph in Figure 2(b), are also reported. (Bottom rows) Typical values of the $P_{o f f}, P_{o s c}, t_{c P}$, and $H_{P}$ of the sinusoidal instability $P(t)=P_{\text {off }}+P_{\text {osc }} \sin \left(\frac{t-t_{c P}}{H_{P}}\right)$ in Equation (6) of the IR power $P(t)$ in the 50 hours following the start of the illumination of the power-meter sensor with IR radiation from the globar source. The parameters $t_{c P}$ and $H_{P}$ are labelled in Figure 2(d). The unit for time in the bottom rows of this table is the hour ([h]).

\begin{tabular}{cccccc}
\hline Time interval & $P_{0}[\mathrm{~mW}]$ & $P_{f}[\mathrm{~mW}]$ & $\tau_{p}[\mathrm{~s}]$ & $\rho_{P}\left[\mathrm{~s}^{-1}\right]$ & $A_{p}[\mathrm{~mW}]$ \\
\hline $0-100 \mathrm{~s}$ & -21.9 & 21.9 & 0.5 & 2.04 & 21.5 \\
\hline Time interval & $P_{\text {off }}[\mathrm{mW}]$ & $P_{\text {osc }}[\mathrm{mW}]$ & $H_{p}[\mathrm{~h}]$ & $t_{c p}[\mathrm{~h}]$ & - \\
\hline $0-50 \mathrm{~h}$ & $21.7 \pm 0.7$ & $0.05 \pm 0.03$ & $4.0 \pm 0.5$ & $16 \pm 2$ & - \\
\hline
\end{tabular}


$\sigma(\boldsymbol{r}, t)$ of the electrons on the illuminated Cu plate of the PG device, and contributes to producing $\Delta V(t)$ through the $\boldsymbol{E}$ and $\boldsymbol{H}$ fields (electric contribution) and $\Delta T(t)$ (entropic contribution).To prove that a link exists between $P(t)$ and $\Delta V(t)$, possibly also between $P(t)$ and $\Delta T(t)$, we sketch the behavior of $\sigma(\boldsymbol{r}, t)$ and relate it to the observed $P(t), \Delta V(t)$, and $\Delta T(t)$.

To sketch $\sigma(\boldsymbol{r}, t)$, we hypothesize that, while hitting the surface of the Cu plate, the IR radiation modulates the electric field $\boldsymbol{E}$ through the sinusoidal instability of the IR power $P(t)=a|\boldsymbol{S}(t)|=a|\boldsymbol{E}(t) \times \boldsymbol{H}(t)|$. In turn, $\boldsymbol{E}$ and its modulation act on the electrons of the Cu plate with force $\boldsymbol{f}=e \boldsymbol{E}$, where $e$ is the electron's charge. As in the photoelectric effect [3], $\boldsymbol{f}$ displaces the electrons away from the location in which the IR radiation impinges on the $\mathrm{Cu}$ plate, locally decreasing their surface density such that $\sigma(\boldsymbol{r}, t) \propto 1 / P(t)$. However, unlike in the photoelectric effect, $\boldsymbol{f}$ does not kick the electrons out of the Cu plate. In this process, $\sigma(\boldsymbol{r}, t)$ varies in time $t$ as well as in space $\boldsymbol{r}$, i.e. the 2-dimensional (2D) surface of the Cu plate. To allow us versatility in choosing reference system, orientation and phase, we represent the 2D space variable $\boldsymbol{r}$ as the complex variable $\mathbf{z}=r_{x}+i r_{y}$, where $i$ is the imaginary unit. This choice resembles that adopted to describe light polarization through Jones matrices [23]-[25]. Thus, $\mathbf{z}=\left[\begin{array}{l}r_{x} \\ r_{y}\end{array}\right]=\left[\begin{array}{l}r_{0 x} \mathrm{e}^{i \varphi_{x}} \\ r_{0 y} \mathrm{e}^{i \varphi_{y}}\end{array}\right]=\left[\begin{array}{l}r_{a}+i r_{b} \\ r_{c}+i r_{d}\end{array}\right]$. All possible rotations of the reference system, phases, and positions in the 2D plane can be obtained by selecting magnitude and sign of $r_{a}, r_{b}$, $r_{c}$, and $r_{d}$.

With this choice of $\mathbf{z}$, upon starting the illumination, we picture $\sigma(\mathbf{z}, t)$ to exponentially decrease according to $\sigma(\mathbf{z}, t)=R E\left(\left|\sigma_{0} \mathrm{e}^{\frac{-t}{\tau_{\sigma}+\left(r_{x}+i r_{y}\right) k+\varphi}}-\right| \sigma_{f} \mid\right)$, where $\sigma_{0}$ and $\sigma_{f}$ are the initial and final surface electron densities, $\tau_{\sigma}$ the time constant, $\boldsymbol{k}$ a vector with units of inverse length, and $\varphi$ an arbitrary phase. We note that the exponential behavior is modulated by the oscillatory function $\mathrm{e}^{i_{y} \mathrm{k}}$.

In the subsequent 30 hours, from Equation (6) we expect $\sigma(\mathbf{z}, t)$ to undergo a slow variation in time such that $\sigma(z, t) \propto 1 / P(t) \propto 1 / \sin \left(\frac{t-t_{c P}}{H_{P}}\right)$, where the sine function has the frequency $v_{P}=1 / 4 H_{P}$ derived in Section 3(a). With the choice of $\mathbf{z}$ discussed above, and utilizing the laws of trigonometric functions for complex variables, we obtain:

$$
\sigma(\mathbf{z}, t) \propto R E\left(\frac{1}{\sin (\mathbf{z}, t)}\right)=R E\left(\frac{1}{\sin \left(r_{x}+i r_{y}, t\right)}\right)=\frac{1}{\sin \left(r_{x}, t\right) \cosh \left(r_{y}, t\right)}=\frac{\operatorname{sech}\left(r_{y}, t\right)}{\sin \left(r_{x}, t\right)}=\frac{\operatorname{sech}\left(\frac{v_{y} t-v_{y} t_{\sigma y}}{L_{y}}\right)}{\sin \left(\frac{v_{x} t-v_{x} t_{\sigma x}}{L_{x}}\right)}
$$

Here, $v_{x}$ and $v_{y}$ are the instability's propagation velocities along the $x$ and $y$ directions; $L_{x}$ and $L_{y}$ are the lengths of the Cu plate along $x$ and $y$; finally, $t_{\sigma x}$ and $t_{\sigma y}$ are the critical times of the surface electron density's instability along $x$ and $y$. Considering $\sigma_{o f f}$ and $\sigma_{o s c}$, the equilibrium electron density and its deviation from equilibrium, respectively, we obtain $\sigma(\mathbf{z}, t)=\sigma_{\text {off }}+\sigma_{\text {osc }}\left(\frac{\operatorname{sech}\left(\frac{v_{y} t-v_{y} t_{\sigma y}}{L_{y}}\right)}{\sin \left(\frac{v_{x} t-v_{x} t_{\sigma x}}{L_{x}}\right)}\right)$. The 2D space variable $\mathbf{z}$, therefore unveils a hyperbolic instability in $\sigma(\mathbf{z}, t)$ modulated by a sine function.

While $\sigma(z, t)$ requires a spatiotemporal set of variables, the functions $\Delta V(t), T_{I R}(t), T_{\text {no-IR }}(t)$, and $\Delta T(t)$ are only time-dependent. To decouple $\mathbf{z}$ from the effects of $\sigma(\mathbf{z}, t)$ on $\Delta V(t), T_{I R}(t), T_{n o-I R}(t)$, and $\Delta T(t)$, and allow time t to be the only effective variable, we integrate $\sigma(z, t)$ over the surface area $a$ of the Cu plate as $\int_{a} \sigma(\mathbf{z}, t) \mathrm{d} \mathbf{z}$. Because of the capacitor-type structure of the PG device, with overall capacitance C, we expect $\Delta V(t)=\frac{a \int_{a} \sigma(\mathbf{z}, t) \mathrm{d} \mathbf{z}}{C}$. This integration causes the loss of correlation between the phase of 
$P(t)$ and $\Delta V(t)$. In the 30 hours after starting the illumination, since $1 / \sin \left(r_{x}, t\right)>1$ where $\left|\sin \left(r_{x}, t\right)\right| \neq 0$, from Equation (7) we expect the $\operatorname{sech}\left(r_{y}, t\right)$ function to determine the behavior in time of $\Delta V(t)$. We also envision the capacitor-type structure of the PG device to affect $T_{I R}(t)$ and $T_{n o-I R}(t)$, while leaving $\Delta T(t)$ constant.

Summarizing, we expect $\Delta V(t)$ to obey an exponential behavior in the first minutes after starting the illumination, and to exhibit a hyperbolic-secant-type of instability in the 30 hours thereafter, with no phase relationship with $P(t)$, no periodic behavior, and with $\Delta T(t)$ constant. We are currently exploring this hypothesis further numerically, which will be the focus of future work.

c) Behavior of $\Delta V(t)$ and $\Delta T(t)$ in the 400 seconds after starting the illumination

The voltage difference $\Delta V(t)$ and temperature difference $\Delta T(t)$ in the 400 seconds after starting the illumination of the PG device with IR radiation are pictured in Figure 3 and Figure 4 for the away and the toward architectures. We find that the voltage difference $\Delta V(t)$ in Figure 3(a) and Figure 3(d) fits a sum of exponential functions:

$$
\Delta V(t)=\sum_{i=1}^{N}\left(\Delta V_{0 i} \mathrm{e}^{-\frac{t}{\tau_{i}}}+\Delta V_{f i}\right)
$$

where the $\Delta V_{0 i}$ and $\Delta V_{f i}$ terms are the initial and final voltage differences, and the $\tau_{i}$ terms are the time constants. These parameters and the number of summands $N$ are summarized in Table 3. In both the away and the toward architectures, the electric contribution is related to the summand with $N=1$ and $\tau_{1}=3.8 \mathrm{~s}$. The
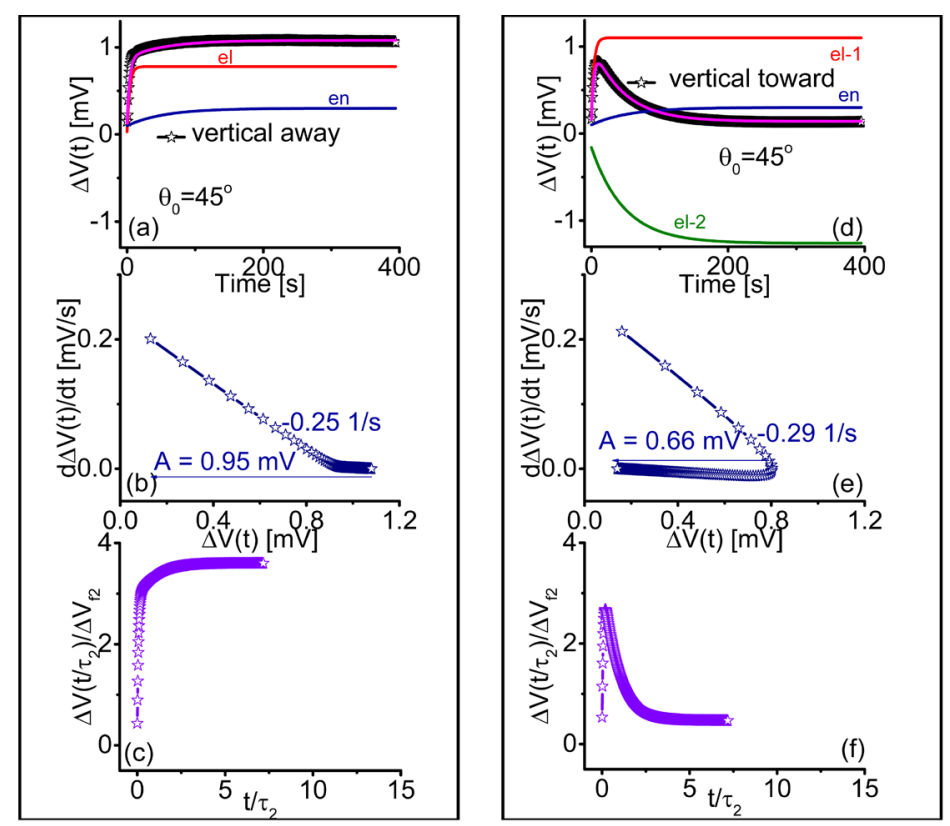

Figure 3. Panels (a), (b), and (c) correspond to the away architecture and refer to the 400 seconds immediately following the start the illumination of the PG device with IR radiation. (a) Voltage difference $\Delta V(t)$ with fitting curves obeying Equation (8) highlighting the summands related to the electric (el) and the entropic (en) contributions; (b) Graph of $\left(\Delta V(t), \frac{\mathrm{d} \Delta V(t)}{\mathrm{d} t}\right)$ obtained from the fitting parameters in Table 3, reporting the slope and amplitude $A$; (c) Dimensionless voltage $\overline{\Delta V(\mathrm{~T})}=\Delta V(t) / \Delta V_{f 2}$ as in Equation (9). Panels (d), (e), and (f) report the voltage difference $\Delta V(t)$, the $\left(\Delta V(t), \frac{\mathrm{d} \Delta V(t)}{\mathrm{d} t}\right)$ graph with slope and amplitude $A$, and the dimensionless voltage $\overline{\Delta V(\mathrm{~T})}=\Delta V(t) / \Delta V_{f 2}$, respectively, for the toward architecture in the 400 seconds immediately following the start the illumination of the PG device with IR radiation. Panel (d) highlights the two summands related to the electric (el-1 and el-2) contributions, and the summand related to the entropic (en) contribution. 


\section{A. L. Gordon et al.}

entropic contribution is related to the summand with larger time constant, $\tau_{2}=55 \mathrm{~s}$, and is labeled with $N=2$. This association is justified because $\tau_{2}=55 \mathrm{~s}$ is of the same order of magnitude of $\tau_{T 1}=30 \mathrm{~s}$ for the temperature difference $\Delta T(t)$ in both architectures, as can be seen in Table 3, and further in Appendix-1. The summand with $N=3$, detected only in the toward architecture, is related to an electric contribution because the "decay" in $\Delta V(t)$, shown in Figure 3(d), is absent in the corresponding $\Delta T(t)$ in Figure 4(c). We claim that the summand with $N=3$ causes the "decay" in $\Delta V(t)$ shown in Figure 3(d) because of the non-continuity of the Cu plate opposite to the IR radiation illustrated in Figure 1(b). The large value of $\Delta V_{03}=1.1 \mathrm{mV}$ signals that the toward architecture tries to avoid the "decay" by pumping up the voltage production.

The rates of increase of $\Delta V(t) \quad\left(\rho_{\Delta V}\right)$ in the initial linear regime of the $\left(\Delta V(t), \frac{\mathrm{d} \Delta V(t)}{\mathrm{d} t}\right)$ graph in Figure 3(b) and Figure 3(e), are $\rho_{\Delta V}=0.25 \mathrm{~s}^{-1}$ in the away architecture, and $\rho_{\Delta V}=0.29 \mathrm{~s}^{-1}$ in the toward architecture. The amplitudes $A$, also derived from Figure 3(b) and Figure $3(\mathrm{e})$, are $0.95 \mathrm{mV}$ and $0.66 \mathrm{mV}$ in the away and the toward architectures, respectively.

Table 3. Fitting parameters $\tau_{i}, \Delta V_{0 i}$, and $\Delta V_{f i}$ of the voltage difference $\Delta V(t)$ in: $\Delta V(t)=\sum_{i=1}^{N}\left(\Delta V_{0 i} \mathrm{e}^{-\frac{t}{\tau_{i}}}+\Delta V_{f i}\right)$, Eq-
uation (8) (top rows) and $\tau_{T i}, \Delta T_{0 i}$, and $\Delta T_{f i}$ of the temperature difference $\Delta T(t)$ in $\Delta T(t)=\sum_{i=1}^{M}\left(\Delta T_{0 i} \mathrm{e}^{-\frac{t}{\tau_{T i}}}+\Delta T_{f i}\right)$, Equation (10) (bottom rows) in the away and toward architectures in the 400 seconds immediately following the start of the illumination of the PG device with IR radiation. The corresponding experimental data are shown in Figure 3(a) and Figure 3(d), and Figure 4(a) and Figure 4(c). The indexes $N$ and $M$ indicate the number of summands in Equations (8) and (10), respectively. The relationship of the summands with either the electric or the entropic contribution is highlighted.

\begin{tabular}{|c|c|c|c|}
\hline $\begin{array}{c}\text { Away } \\
\Delta V ; \quad N=2\end{array}$ & Figure 3(a): $N=1$, electric & Figure 3(a): $N=2$, entropic & - \\
\hline & $\tau_{1}=3.8 \mathrm{~s}$ & $\tau_{2}=55.0 \mathrm{~s}$ & - \\
\hline & $\Delta V_{01}=-0.75 \mathrm{mV}$ & $\Delta V_{02}=-0.20 \mathrm{mV}$ & - \\
\hline & $\Delta V_{f 1}=0.78 \mathrm{mV}$ & $\Delta V_{f 2}=0.30 \mathrm{mV}$ & - \\
\hline \multirow[t]{4}{*}{$\begin{array}{c}\text { Toward } \\
\Delta V ; \quad N=3\end{array}$} & Figure 3(d): $N=1$, electric & Figure 3(d): $N=2$, entropic & Figure 3(d): $N=3$ electric \\
\hline & $\tau_{1}=3.8 \mathrm{~s}$ & $\tau_{2}=55.0 \mathrm{~s}$ & $\tau_{3}=48.0 \mathrm{~s}$ \\
\hline & $\Delta V_{01}=-0.88 \mathrm{mV}$ & $\Delta V_{02}=-0.20 \mathrm{mV}$ & $\Delta V_{03}=1.10 \mathrm{mV}$ \\
\hline & $\Delta V_{f 1}=1.10 \mathrm{mV}$ & $\Delta V_{f 2}=0.30 \mathrm{mV}$ & $\Delta V_{f 3}=-1.26 \mathrm{mV}$ \\
\hline \multirow[t]{4}{*}{ Away $\Delta T ; M=1$} & Figure 4(a): $\quad M=1$, entropic & - & - \\
\hline & $\tau_{T 1}=30.0 \mathrm{~s}$ & - & - \\
\hline & $\Delta T_{01}=-0.135^{\circ} \mathrm{C}$ & - & - \\
\hline & $\Delta T_{f 1}=-0.26^{\circ} \mathrm{C}$ & - & - \\
\hline \multirow[t]{4}{*}{$\begin{array}{c}\text { Toward } \\
\Delta T ; \quad M=2\end{array}$} & Figure 4(c): $M=1$, entropic & Figure 4(c): $M=2$, entropic & - \\
\hline & $\tau_{T 1}=30.0 \mathrm{~s}$ & $\tau_{T 2}=2000.0 \mathrm{~s}$ & - \\
\hline & $\Delta T_{01}=-0.136^{\circ} \mathrm{C}$ & $\Delta T_{02}=-0.36^{\circ} \mathrm{C}$ & - \\
\hline & $\Delta T_{f 1}=-0.25^{\circ} \mathrm{C}$ & $\Delta T_{f 2}=0.20^{\circ} \mathrm{C}$ & - \\
\hline
\end{tabular}




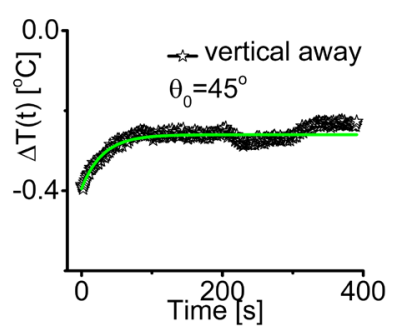

(a)

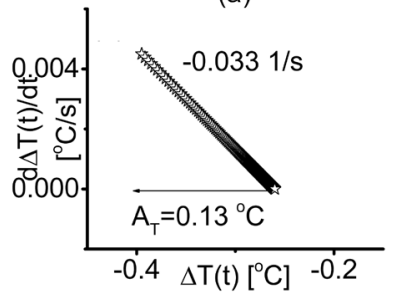

(b)

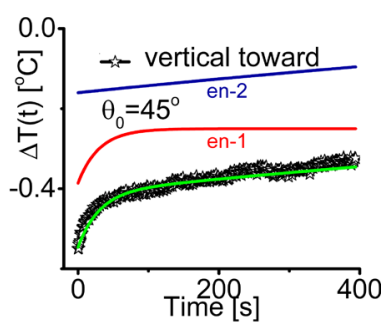

(c)

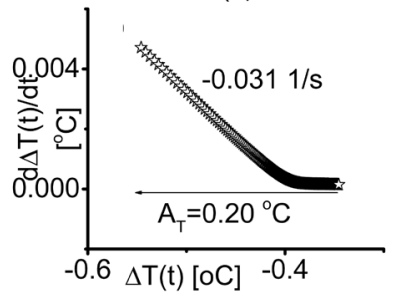

(d)

Figure 4. Panels (a) and (b) correspond to the away architecture and refer to the 400 seconds immediately following the start of the illumination of the PG device with IR radiation. (a) Temperature difference $\Delta T(t)$ with exponential behavior as in Equation (10); (b) Graph of $\left(\Delta T(t), \frac{\mathrm{d} \Delta T(t)}{\mathrm{d} t}\right)$ obtained from fitting parameters in Table 3, reporting the slope and amplitude $A_{T}$. Panels (c) and (d) report the temperature difference $\Delta T(t)$ and the $\left(\Delta T(t), \frac{\mathrm{d} \Delta T(t)}{\mathrm{d} t}\right)$ graph with slope and amplitude $A_{T}$ for the toward architecture in the 400 seconds immediately following the start the illumination of the PG device with IR radiation. Panel (c) highlights the two summands related to the entropic (en-1 and en-2) contribution.

As with the power $P(t)$, the voltage difference $\Delta V(t)$ produced by the PG device also rises exponentially in the first few minutes after starting the illumination. However, $\rho_{\Delta V}=0.25$ and $0.29 \mathrm{~s}^{-1}$ are one order of magnitude smaller than $\rho_{P}=2.04 \mathrm{~s}^{-1}$ for the power $P(t)$ shown in Figure 2(b). We ascribe this discrepancy to the activation process occurring in the PG device to start the $\Delta V(t)$ production.

We capture the relative behavior of the electric and the entropic contributions to the generation of $\Delta V(t)$ using the dimensionless voltage $\overline{\Delta V(\mathrm{~T})}=\Delta V(t) / \Delta V_{f 2} \quad$ [22], pictured in Figure 3(c) and Figure 3(f), and derived from Equation (8) and its parameters in Table 3 as follows:

$$
\widehat{\Delta V(\mathrm{~T})}=\sum_{i=1}^{N}\left(\frac{\Delta V_{0 i}}{\Delta V_{f 2}} \mathrm{e}^{-\mathrm{T} \frac{\tau_{2}}{\tau_{i}}}+\frac{\Delta V_{f i}}{\Delta V_{f 2}}\right)
$$

Here, the term $\mathrm{T}=t / \tau_{2}$ is the dimensionless time [22], while $r_{0 i}=\Delta V_{0 i} / \Delta V_{f 2}$ and $r_{f i}=\Delta V_{f i} / \Delta V_{f 2}$ are dimensionless voltage parameters. We performed the normalization with respect to the slower summand, i.e. the one related to the entropic contribution with $N=2$. Such a choice leads us to observe an evolution of $\overline{\Delta V(\mathrm{~T})}$ in Figure 3(c) and Figure 3(f) on a similar T-scale in both the away and the toward architectures, signifying that the dynamics is not affected by the architecture. On the other hand, from the plateau value at $\overline{\Delta V(T)} \approx 4$ in the away architecture, we infer that the summands related to the electric and entropic contributions add one to the other, with the former prevailing over the latter because of $r_{f 1}=\Delta V_{f 1} / \Delta V_{f 2}=2.6>1$. On the contrary, from the plateau value at $\overline{\Delta V(\mathrm{~T})}<1$ in the toward architecture, we conclude that the two summands related to the electric contributions with $N=1$ and 3 almost annihilate one another, while the summand related to the entropic contribution survives in the long term.

The temperature difference $\Delta T(t)$, shown in Figure 4(a) and Figure 4(c), surprisingly exhibits negative values, indicating that $T_{I R}(t)<T_{n o-I R}(t)$. This behavior is typical when the PG device faces the IR radiation with the Cu plate. Nevertheless, we find that the temperature difference $\Delta T(t)$ rises exponentially as follows: 


$$
\Delta T(t)=\sum_{i=1}^{M}\left(\Delta T_{0 i} \mathrm{e}^{-\frac{t}{\tau_{T i}}}+\Delta T_{f i}\right),
$$

where $\Delta T_{0 i}$ and $\Delta T_{f i}$ are the initial and final temperature differences, respectively, and the $\tau_{T i}$ terms are the time constants. These time constants reflect the fact that generally $T_{I R}(t)$ rises faster than $T_{\text {no-IR }}(t)$. The parameters and the number of summands $M$, all related to the entropic contribution, are summarized in Table 3 . We observe that $1 \leq M \leq 2$, and that summands similar to that with $M=2$ (rarely detected) in the toward architecture in Figure 4(c) exhibit an extremely large time constant of $\tau_{T 2}=2000 \mathrm{~s}$. We also note that no "decay" appears for $\Delta T(t)$ in the toward architecture in Figure 4(c), as opposed to the corresponding voltage difference $\Delta V(t)$ in Figure $3(\mathbf{d})$.

The slopes of the initial linear regimes in the $\left(\Delta T(t), \frac{\mathrm{d} \Delta T(t)}{\mathrm{d} t}\right)$ graphs, displayed in Figure $4(\mathbf{b})$ and Figure 4(d), are negative, as those of $P(t)$ in Figure 2(b), and $\Delta V(t)$ in Figure 3(b) and Figure 3(e). The rates of increase of $\Delta T(t)$ are $\rho_{\Delta T}=0.033 \mathrm{~s}^{-1}$ and $\rho_{\Delta T}=0.031 \mathrm{~s}^{-1}$ in the away and toward architectures, respectively. The corresponding amplitudes $A_{T}$ are $0.13^{\circ} \mathrm{C}$ and $0.20^{\circ} \mathrm{C}$ in the away and the toward architectures, respectively. The ratio $R=\Delta(\Delta V) / \Delta(\Delta T)$ between the jump in voltage $(\Delta(\Delta V))$ and temperature difference $(\Delta(\Delta T))$ in the away architecture is $R=7.31 \mathrm{mV} /{ }^{\circ} \mathrm{C}$.

Overall, Figure 3 and Figure 4 suggest that $\Delta V(t)$ and $\Delta T(t)$ evolve independently, with different rates of increase such that $\rho_{\Delta V} \gg \rho_{\Delta T}$. The electric contribution affects only $\Delta V(t)$. Thus, we conclude that the electric and entropic contributions in the interaction of IR radiation with a PG device are decoupled. To further support this conclusion, in Appendix-1 we will compare the trends of $\rho_{\Delta V}, \rho_{\Delta T}, R=\Delta(\Delta V) / \Delta(\Delta T)$, and the values of $\Delta(\Delta V)$ upon activating the PG device with IR radiation, as done so far, and conductive heat transfer from a $100 \Omega$ resistor and a 0.02 A current. Only the entropic contribution is activated in this case because the produced power of $0.04 \mathrm{~W}$, corresponding to a temperature of $\approx 24^{\circ} \mathrm{C}$ on the PG device, is too low to produce significant blackbody radiation to trigger the electric contribution.

d) Behavior of $\Delta V(t)$ and $\Delta T(t)$ in the 30 hours after starting the illumination

The voltage difference $\Delta V(t)$ and temperature difference $\Delta T(t)$ evolve in the 30 hours after starting the illumination of the PG device as pictured in Figure 5 and Figure 6. We observed one possible behavior in Figure 5, which displays the data collected with the toward architecture. The voltage difference $\Delta V(t)$ in Figure 5(a), exhibits an instability around the $20^{\text {th }}$ hour. No instability is detected in $\Delta T(t)$ in Figure 5(b), which is almost flat. The same instability affecting $\Delta V(t)$, however, is revealed in $T_{I R}(t)$ and $T_{\text {no-IR }}(t)$, displayed in Figure 5(c) and Figure 5(d), respectively. This finding suggests that a strong correlation exists among $\Delta V(t)$, $T_{I R}(t)$ and $T_{n o-I R}(t)$, which we ascribe to the capacitor-type of structure of the PG device, as predicted in Section 3(b).

We observed another possible behavior in Figure 6, displaying data collected with the away architecture. In this case, $\Delta V(t)$, shown in Figure 6(a), exhibits an instability around the $12^{\text {th }}$ hour which is uncorrelated with the trends occurring in $T_{I R}(t)$ and $T_{\text {no-IR }}(t)$. The lack of correlation highlights the lack of coupling between the electric and the entropic contributions. Since the trends of the two temperatures are identical, we show only $T_{I R}(t)$ in Figure 6(b). We omit to display $\Delta T(t)$, which is flat as hypothesized in Section 3(b). The instability of $\Delta V(t)$ in Figure 6(a) is non-periodic, as predicted in Section 3(b). Thus, to find a suitable fitting function, we recall the hypothesis in Section 3(b) where we pictured the time-dependence of $\Delta V(t)$ in Figure 6(a) as captured by a hyperbolic secant function with no phase relationship with $P(t)$ and no sinusoidal periodicity. Thus, we propose:

$$
\Delta V(t)=\Delta V_{\text {off }}+\Delta V_{\text {osc }} \operatorname{sech}\left(\frac{t-t_{c}}{H}\right)
$$

In this expression, $\Delta V_{\text {off }}$ and $\Delta V_{\text {osc }}$ are the offset and amplitude of the departure of $\Delta V(t)$ from the offset, respectively. The positive or negative sign of $\Delta V_{\text {osc }}$ corresponds to a downward or upward concavity, respectively, of the instability. The critical time $t_{c}$ is the instant in which the maximum $\left|\Delta V_{\text {osc }}\right|$ value is achieved. Finally, the term $H$ indicates the half width at half maximum (HWHM), or minimum (depending upon the sign of $\Delta V_{\text {osc }}$ ) of the instability. The magnitude of $\Delta V_{\text {off }}$ corresponds to the long term equilibrium voltage 


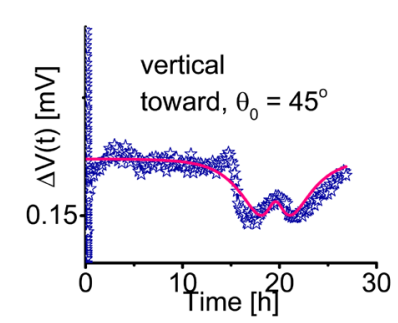

(a)

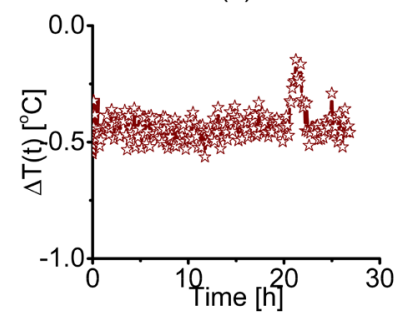

(b)

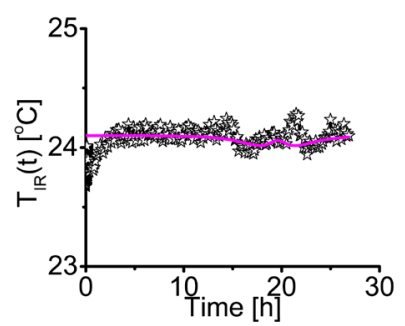

(c)

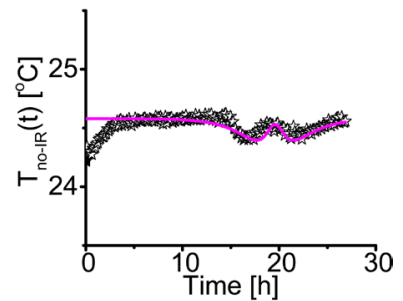

(d)

Figure 5. Panels (a), (b), (c), and (d) correspond to data collected from the toward architecture in the whole time span of about 30 hours following the start of the illumination of the PG device with IR radiation. (a) Voltage difference $\Delta V(t)$. (b) Temperature difference $\Delta T(t)$. Temperatures $T_{I R}(t)$ (c) and $T_{n o-I R}(t)$ (d) of the illuminated and non-illuminated faces, respectively, of the PG device. The zero of the time-scale coincides with the start of the illumination with the IR radiation, and the unit for time is the hour ([h]).

$\Delta V_{f 2}$ reported in Table 3 . The typical values of $\Delta V_{\text {off }}, \Delta V_{\text {osc }}, t_{c}$, and $\mathrm{H}$ are reported in Table 4, labelled in Figure 6(a), and were obtained by placing the zero of the time-scale at the start of the illumination. We highlight that, since $T_{I R}(t)$ in Figure 6(b) does not peak at $t_{c}$, it does not correlate with the behavior in time of $\Delta V(t)$. The instability in $\Delta V(t)$ is evidenced in the $\left(\Delta V(t), \frac{\mathrm{d} \Delta V(t)}{\mathrm{d} t}\right)$ graph in Figure 6(c), where stable and unstable fixed points [20] alternate in a complex fashion without periodicity.

Since the time-dependence is enclosed in a hyperbolic secant function, we name the instability in $\Delta V(t)$ in Equation (11) and Figure 6(a) as hyperbolic instability. We establish the lack of correlation between $\Delta V(t)$, on one hand, and $T_{I R}(t)$ and $T_{\text {no-IR }}(t)$ on the other, as the criterion to identify such instability. Since the hyperbolic instability in $\Delta V(t)$ is absent in $\Delta T(t)$, as expected from Section 3(b), we relate the instability to the sole electric contribution.

e) Correlation between $P(t)$ and $\Delta V(t)$ in the 30 hours after starting the illumination

Here we highlight the correlations existing between the power $P(t)$ and the voltage difference $\Delta V(t)$ to further support the choice of the fitting function in Equation (11) for $\Delta V(t)$ based on the hypothesis highlighted in Section 3(b).

1) The average $H_{P}$ of the power $P(t)$ in Table 2 is $4.0 \pm 0.5 \mathrm{~h}$, about twice the value of $1.82 \mathrm{~h}$ reported in Table 4 for $\mathrm{H}$ of $\Delta V(t)$.

2) The instabilities in $P(t)$ and $\Delta V(t)$ are both small: the former is $P_{\text {off }} / P_{o s c} \approx 10^{3}$, while the latter is $\Delta V_{\text {off }} / \Delta V_{\text {osc }} \approx 10 / 10^{2}$.

3) The $t_{c}$ term in Table 4 is found at $11.65 \mathrm{~h}$, which is reasonably close to the average $t_{c P}$ at $16 \pm 2$ in Table 2.

These observations further support our hypothesis in Section 3(b) relating the sinusoidal instability of $P(t)$ to $\Delta V(t)$ through $\sigma(\mathbf{z}, t)$ on the $\mathrm{Cu}$ plate. In Appendix-2 we will show that the hyperbolic instability in $\Delta V(t)$, selected according to the criterion established in Section 3(d), occurs frequently in our observations. We will provide further evidence of the link between $t_{c}$ and $t_{c P}$. Finally, we will prove the lack of phase relationship between $P(t)$ and $\Delta V(t)$ through lack of predictability of the sign of the amplitude $\Delta V_{\text {osc }}$ of the departure of $\Delta V(t)$ from equilibrium.

f) The nonlinearity of the hyperbolic instability in $\Delta V(t)$

We found that Equation (11), used to fit the hyperbolic instability in $\Delta V(t)$ in the 30 hours after starting the 


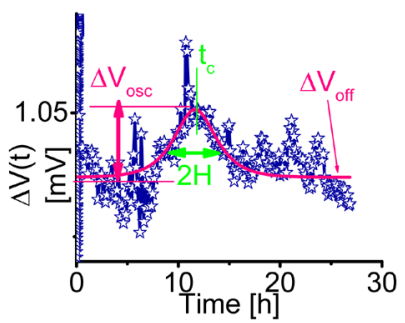

(a)

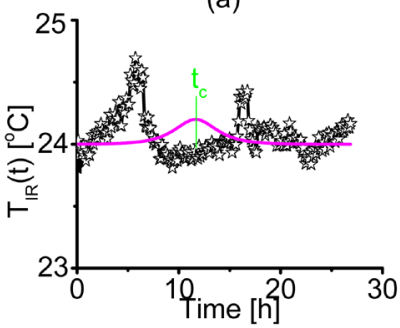

(b)

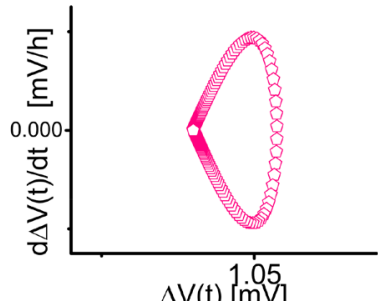

(c)

Figure 6. Panels (a), (b), and (c) correspond to data collected from the away architecture in the whole time span of about 30 hours following the start of the illumination of the PG device with IR radiation. (a) Voltage difference $\Delta V(t)$ exhibiting the hyperbolic instability obeying Equation (11). The parameters $\Delta V_{\text {off }}, \Delta V_{\text {osc }}, t_{c}$, and $H$, reported in Table 4, are labelled. (b) Temperature $T_{I R}(t)$ of the face of the PG device illuminated by the IR radiation, and exhibiting no correlation with the hyperbolic instability in $\Delta V(t)$ highlighted by $t_{c}$. (c) Graph of $\left(\Delta V(t), \frac{\mathrm{d} \Delta V(t)}{\mathrm{d} t}\right)$ obtained using the parameters in Table 4 . The zero of the time-scale coincides with the start of the illumination with the IR radiation, and the unit for time is the hour ([h]).

Table 4. Parameters of $\Delta V(t)=\Delta V_{\text {off }}+\Delta V_{\text {osc }} \operatorname{sech}\left(\frac{t-t_{c}}{H}\right)$ in Equation (11) used to fit the voltage difference $\Delta V(t)$ in Figure 6(a) collected from the away architecture in the time interval of about 30 hours following the start the illumination of the PG device with IR radiation. The parameters $\Delta V_{\text {off }}, \Delta V_{\text {osc }}, t_{c}$, and $H$ are labeled in Figure 6(a).

\begin{tabular}{ccccc}
\hline Away & $\Delta V_{\text {off }}[\mathrm{mV}]$ & $\Delta V_{\text {osc }}[\mathrm{mV}]$ & $t_{c}[\mathrm{~h}]$ & $H[\mathrm{~h}]$ \\
\hline 1.037 & 0.0137 & 11.65 & 1.82 \\
\hline
\end{tabular}

illumination of the PG device with IR radiation, can be a solution of the equation:

$$
\sigma \Delta V(t) \frac{\partial \Delta V(t)}{\partial t}+\varsigma \frac{\partial^{3} \Delta V(t)}{\partial t^{3}}=0
$$

Equation (12) is nonlinear because of the $3^{\text {rd }}$ order partial differential of $\Delta V(t)$ with respect to time $t$. The equation resembles the Korteweg-de Vries (KdV) equation [26] [27] after eliminating the space-dependence, and flipping the space variable with time $t$. In order for $\Delta V(t)$ in Equation (11) to be a solution of Equation (12), we select the $\sigma$ and $\varsigma$ coefficients by rewriting Equation (12) as: 


$$
\Omega \Delta V(t) \frac{\partial \Delta V(t)}{\partial t}+\frac{\partial^{3} \Delta V(t)}{\partial t^{3}}=0
$$

By substituting Equation (11) into Equation (13), we obtain:

$$
\Omega(t)=\frac{1}{H^{2}}\left(\frac{5 \operatorname{sech}\left(\frac{t-t_{c}}{H}\right)^{2}-\tan \left(\frac{t-t_{c}}{H}\right)^{2}}{\Delta V_{\text {osc }} \operatorname{sech}\left(\frac{t-t_{c}}{H}\right)+\Delta V_{\text {off }}}\right) .
$$

The time-dependent coefficient $\Omega(t)=\sigma(t) / \varsigma(t)$ of Equation (13) stands for nonlinear effects [26], and infers that a complex dynamics is hidden in the hyperbolic instability of $\Delta V(t)$ [28] [29]. In Figure 7 we graph $\Omega(t)$ using the parameters reported in Table 4 for the away architecture. Despite the complexity of Equation (14), $\Omega(t)$ appears surprisingly simple, correlates with the time-scale of the hyperbolic instability in $\Delta V(t)$, and peaks at $t_{c}$. Furthermore, we determined the physical units of $\Omega(t)$ to be $1 / h^{2}(1 / \mathrm{mV}$ ) (where $h$ indicates the hour), which suggests that $\Omega(t)$ could be interpreted as the change in time of the rate of $1 / \Delta V(t)$, or simply, the "acceleration" of the inverse voltage. Such identification points out the existence of nonlinear "forces" that continuously push and slow down the changes in $\Delta V(t)$. We plan on further investigating this topic.

\section{Summary and Significance}

We identify and decouple an electric and an entropic contribution to energy transfer from low power infrared radiation to a power generator device. The electric contribution is related to the effects of the electric $\boldsymbol{E}$ and magnetic $\boldsymbol{H}$ fields in the infrared radiation and is detected through the voltage produced by the power generator device. The entropic contribution is mainly related to the temperature difference between the faces of the device. Two observations enable us to decouple the electric and entropic contributions. First, the electric contribution imparts faster rates of increase of the voltage difference $\Delta V(t)$ produced by the power generator device immediately after starting the illumination with infrared radiation. Second, the electric contribution generates a hyperbolic instability in the 30 hours after starting the illumination. The entropic contribution changes slowly and simply reflects the relative delay with which the two faces of the power generator device respond to thermal perturbations.

Our preliminary studies suggest that it is important to learn to exploit the electric contribution, because it offers a variety of ways to increase the voltage produced by the power generator device. For example, blackcolored gaffer tape on the illuminated face doubles the amount of voltage produced, as discussed in Appendix-1, while the temperature difference, thus the entropic contribution, is not sensitive to the presence of the tape. Our findings are relevant for understanding the mechanisms for harvesting IR radiation, and possibly other electromagnetic radiations, through a power generation device as an alternative energy source.

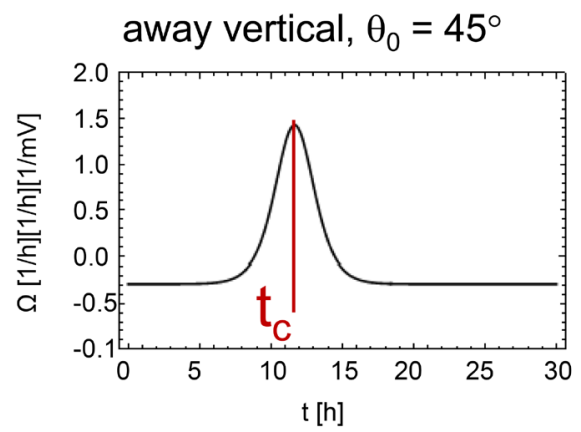

Figure 7. "Acceleration" of the inverse voltage $\Omega(t)$ for the away architecture obtained from Equation (14) using the parameters in Table 4. The time-dependent coefficient $\Omega(t)$ peaks at $t_{c}$, reported in Table 4 . The unit for time is the hour ([h]). 
The future efforts will be devoted to overcome the limitations of the present work: 1) understand the relationship among infrared source power, surface charge density on the power generator, and produced voltage, 2) clarify the behavior of the power generator as a capacitor, and 3) investigate the role of infrared radiation power on the results.

\section{Acknowledgements}

This work was supported by the U.S. Office of Naval Research (awards \# N000141410378 N000141512158), JMU 4-VA Consortium (2013), Thomas F. Jeffress and Kate Miller Jeffress Memorial Trust (grant \# J-1053), the Madison Trust-Fostering Innovation and Strategic Philanthropy-Innovation Grant 2015, the JMU Program of Grants for Faculty Assistance 2014, the JMU Center for Materials Science, and the JMU Department of Physics and Astronomy. The authors thank Dr. A. V. Zenkevich (Moscow Institute of Physics and Technology), Prof. G. Casati (University of Insubria, Italy), and Prof. D. J. Lawrence (JMU) for fruitful discussions.

\section{References}

[1] Christillin, P. (1986) Nuclear Compton Scattering. Journal of Physics G: Nuclear and Particle Physics, 12, 837-851. http://dx.doi.org/10.1088/0305-4616/12/9/008

[2] Siegbahn, K.M. (1981) Electron Spectroscopy for Atoms, Molecules and Condensed Matter. Nobel Lecture, 8 December.

[3] Einstein, A. (1905) Concerning an Heuristic Point of View toward the Emission and Transformation of Light. Annalen der Physik, 17, 132-148. http://dx.doi.org/10.1002/andp.19053220607

[4] Becquerel, E. (1839) Mémoire sur les effets électriques produits sous l'influence des rayons solaires. Comptes Rendus, 9, 561-567.

[5] Burdick, G.A. (1963) Energy Band Structure of Copper. Physical Review, 129, 138-150. http://dx.doi.org/10.1103/PhysRev.129.138

[6] Newnham, R.E., Jang, S.J., Xu, M. and Jones, F. (1991) Fundamental Interaction Mechanisms between Microwaves and Matter. Ceramic Transactions, 21, 51-67.

[7] Kliewer, K.L. and Fuchs, R. (1966) Optical Modes of Vibration in an Ionic Crystal Slab including Retardation. I. Nonradiative Region. Physical Review, 144, 495-503. http://dx.doi.org/10.1103/PhysRev.144.495

[8] Kliewer, K.L. and Fuchs, R. (1966) Optical Modes of Vibration in an Ionic Crystal Slab including Retardation. II. Radiative Region. Physical Review, 150, 573-588. http://dx.doi.org/10.1103/PhysRev.150.573

[9] Fuchs, R., Kliewer, K.L. and Pardee, W.J. (1966) Optical Properties of an Ionic Crystal Slab. Physical Review, 150, 589-596. http://dx.doi.org/10.1103/PhysRev.150.589

[10] Berreman, D.W. (1963) Infrared Absorption at Longitudinal Optic Frequency in Cubic Crystal Films. Physical Review, 130, 2193-2198. http://dx.doi.org/10.1103/PhysRev.130.2193

[11] Gest, H. (2002) History of the Word Photo Synthesis and Evolution of Its Definition. Photosynthesis Research, 73, 710. http://dx.doi.org/10.1023/A:1020419417954

[12] Kuesco, G., Mauer, P.C., Yao, N.Y., Kubo, M., Noh, H.J., Lo, P.K., Park, H. and Lukin, M.D. (2013) Nanometre-Scale Thermometry in a Living Cell. Nature, 500, 54-59. http://dx.doi.org/10.1038/nature12373

[13] Jameson, A.D., Tomaino, J.L., Lee, J.-S., Khitrova, G., Gibbs, H.M., Böttge, C.N., Klettke, A.C., Kira, M. and Koch, S.W. (2014) Direct Measurement of Light-Matter Energy Exchange inside a Microcavity. Optica, 1, 276-280. http://dx.doi.org/10.1364/OPTICA.1.000276

[14] Kumar, A., Low, T., Fung, K.H., Avouris, P. and Fang, N.X. (2015) Tunable Light-Matter Interaction and the Role of Hyperbolicity in Graphene-hBN System. Nano Letters, 15, 3172-3180. http://dx.doi.org/10.1021/acs.nanolett.5b01191

[15] Richter, C.-P., Rajguru, S., Stafford, R. and Stock, S.R. (2013) Radiant Energy during Infrared Neural Stimulation at the Target Structure. Proceedings of SPIE, 8565, Article ID: 85655P. http://dx.doi.org/10.1117/12.2013849

[16] Eisen, D., Janssen, D., Chen, X., Choa, F.-S., Kotsov, D. and Fan, J. (2013) Closing a Venus Flytrap with Electrical and Mid-IR Photon Stimulations. Proceedings of SPIE, 8565, Article ID: 85655I. http://dx.doi.org/10.1117/12.2005351

[17] Tritt, T.M., Böttner, H. and Chen, L. (2008) Thermoelectrics: Direct Solar Thermal Energy Conversion. MRS Bulletin, 33, 366-368. http://dx.doi.org/10.1557/mrs2008.73

[18] Tritt, T.M. (2011) Thermoelectric Phenomena, Materials, and Applications. Annual Review of Materials Research, 41, 433-438. http://dx.doi.org/10.1146/annurev-matsci-062910-100453 
[19] Bell, L.E. (2008) Cooling, Heating, Generating Power, and Recovering Waste Heat with Thermoelectric Systems. Science, 321, 1457-1461. http://dx.doi.org/10.1126/science.1158899

[20] Vining, C.B. (2009) An Inconvenient Truth about Thermoelectrics. Nature Materials, 8, 83-85. http://dx.doi.org/10.1038/nmat2361

[21] Schwab, Y., Mann, H.S., Lang, B.N., Lancaster, J.L., Parise, R.J., Vincent-Johnson, A.J. and Scarel, G. (2013) Infrared Power Generation in an Insulated Compartment. Complexity, 19, 44-55. http://dx.doi.org/10.1002/cplx.21484

[22] Strogatz, S.H. (1994) Nonlinear Dynamics and Chaos. Westview Press, Cambridge, MA.

[23] Jones, R.C. (1941) A New Calculus for the Treatment of Optical Systems. I. Description and Discussion of the Calculus. Journal of the Optical Society of America, 31, 488-493. http://dx.doi.org/10.1364/JOSA.31.000488

[24] Jones, R.C. (1941) A New Calculus for the Treatment of Optical Systems. III. The Sohncke Theory of Optical Activity. Journal of the Optical Society of America, 31, 500-503. http://dx.doi.org/10.1364/JOSA.31.000500

[25] Jones, R.C. (1942) A New Calculus for the Treatment of Optical Systems. IV. Journal of the Optical Society of America, 32, 486-493. http://dx.doi.org/10.1364/JOSA.32.000486

[26] Korteweg, D.J. and de Vries, G. (1895) On the Change of Form of Long Waves Advancing in a Rectangular Canal and a New Type of Long Stationary Waves. Philosophical Magazine Series, 39, 422-443. http://dx.doi.org/10.1080/14786449508620739

[27] Smaoui, N. and Zribi, M. (2009) A Finite Dimensional Control of the Dynamics of the Generalized Korteweg-de Vries Burgers Equation. Applied Mathematics \& Information Sciences, 3, 207-221.

[28] Jiang, Y., Tian, B., Liu, W.-J., Sun, K. and Qu, Q.-X. (2010) Soliton Solutions for a Variable-Coefficient Korteweg-de Vries Equation in Fluids and Plasmas. Physica Scripta, 82, Article ID: 055008. http://dx.doi.org/10.1088/0031-8949/82/05/055008

[29] Vlieg-Hulstman, M. and Halford, W.D. (1995) Exact Solutions to KdV Equations with Variable Coefficients and/or Nonuniformities. Computers \& Mathematics with Applications, 29, 39-47. http://dx.doi.org/10.1016/0898-1221(94)00205-Y

[30] Mann, H.S., Schwab, Y., Lang, B.N., Lancaster, J.L., Parise, R.J. and Scarel, G. (2014) Effective Thermoelectric Power Generation in an Insulated Compartment. World Journal of Condensed Matter Physics, 4, 153-165. http://dx.doi.org/10.4236/wjcmp.2014.43020 


\section{Appendix-1}

To highlight the effects of the entropic contribution and decouple it from the electric contribution, we collected the voltage difference $\Delta V(t)$ and the temperature difference $\Delta T(t)$ from the PG device activated by conductive heat transfer from a $100 \Omega$ resistor in contact with the surface of the device. A temperature of $\approx 24^{\circ} \mathrm{C}$ was generated by the PG device activated with a B\&K Precision 1665 power supply providing $0.02 \mathrm{~A}$ and $3.2 \mathrm{~V}$ to the resistor. The achieved temperature is of the same order of magnitude as that detected when IR radiation hits the PG device, as can be seen in Figure 5(c) and Figure 5(d). We used a PG device 07111-9L31-04B by Custom Thermoelectric Inc. finished with an AlO plate (not with a Cu plate) and, in selected cases, gaffer tape. We placed the sample holders on both sides of the PG device in the toward architecture to create a capacitor structure with electrodes on both faces and thus avoid the "decay" in $\Delta V(t)$ shown in Figure 3(d). The experiments investigating the effects of conductive heat transfer were performed in an insulated sample compartment described in Ref. [30]. The PG device was horizontally fixed on the sample holders in all the measurements. When the IR radiation was used to compare the results obtained with conductive heat transfer, the PG device was also positioned horizontally and at an angle of incidence $\theta_{0}=30^{\circ}$ with respect to the IR radiation. The basic experimental parameters are summarized in Table 1.

In the 400 seconds immediately following the start the activation of the PG device by either conductive heat transfer or IR radiation, we measured the rate of increase of $\Delta V(t)\left(\rho_{\Delta V}\right)$ and $\Delta T(t)\left(\rho_{\Delta T}\right)$, and the ratio $R=\Delta(\Delta V) / \Delta(\Delta T)$ between the jumps in voltage $(\Delta(\Delta V))$ and temperature difference $(\Delta(\Delta T))$ in the away and toward architectures. We report the average values of $\rho_{\Delta V}, \rho_{\Delta T}$, and $R$, alongside their uncertainties, in Figures A1(a)-(c), respectively. Figure A1(a) indicates that the average $\rho_{\Delta V}$ values obtained by activating the PG device with IR radiation are always larger than those obtained by activating the PG device using conductive heat transfer. Figure A1(b) suggests that the average $\rho_{\Delta T}$ values have a common value, independently of the source of activation of the PG device. Moreover, the average $\rho_{\Delta V}$ and $\rho_{\Delta T}$ values are similar when the PG device is activated by conductive heat transfer. In addition, the $\rho_{\Delta T}$ values coincide with those derived from Figure 4(b) and Figure 4(d). This finding supports the identification of the contribution to energy transfer with slower rate of increase with the entropic contribution. Figure A1(c) shows that, within the errors, the average

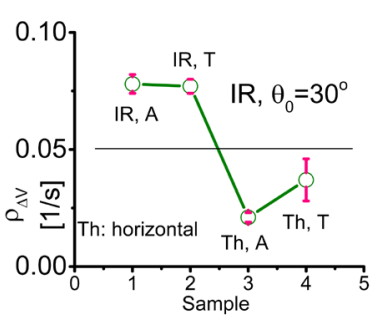

(a)

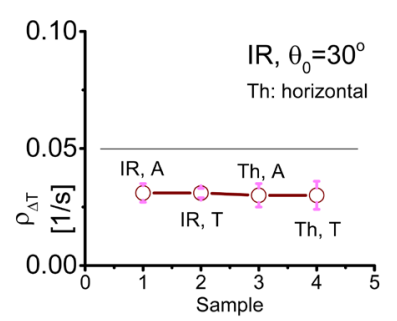

(b)

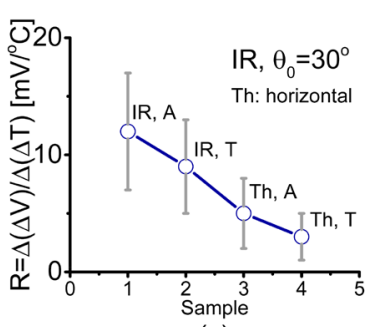

(c)

Figure A1. (a) Rate of increase of the voltage difference ( $\rho_{\Delta V}$ ). (b) Rate of increase of the temperature difference ( $\left.\rho_{\Delta T}\right)$. (c) Ratio $R=\Delta(\Delta V) / \Delta(\Delta T)$ between the jump in voltage $(\Delta(\Delta V))$ and temperature difference $(\Delta(\Delta T))$. The data were obtained performing measurements in the away (A) and toward $(T)$ architectures on a PG device finished with an AlO plate covered with gaffer tape, described in Appendix-1. The results compare data obtained in the 400 seconds immediately following the start the activation of the PG device with IR radiation (IR) or conductive heat transfer (Th) from a $100 \Omega$ resistor. The horizontal line at $0.05 \mathrm{~s}^{-1}$ in panels (a) and (b) highlights the level above which only $\rho_{\Delta V}$ values due to IR radiation can be detected. The PG device was horizontally fixed on the sample holders in all the measurements. When the IR radiation was used, the PG device was positioned at an angle of incidence $\theta_{0}=30^{\circ}$ with respect to the IR radiation. 
value of the ratio $R$ is larger when the PG device is activated by IR radiation through the electric contribution.

In the 400 seconds immediately following the start the activation of the PG device by either conductive heat transfer or IR radiation, we also measured the jump in voltage $(\Delta(\Delta V))$ obtained performing measurements on the PG device described above and finished with colored gaffer tape. We performed the measurements for the away and toward architectures. Figure A2(a) displays the magnitude of $\Delta(\Delta V)$ obtained by activating the PG device with conductive heat transfer from a $100 \Omega$ resistor. The observed trends are neither affected by the color nor by the presence of the tape. On the other hand, Figure A2(b) shows that the magnitude of $\Delta(\Delta V)$ obtained by activating the PG device with IR radiation, is slightly sensitive to the color of the tape, and exhibits a noticeable drop when the tape is absent. Interestingly, the black-colored tape on the illuminated face of the PG device doubles the magnitude of $\Delta(\Delta V)$ compared to the case without tape. Values of $\Delta(\Delta V)$ above $0.5 \mathrm{mV}$, represented by the horizontal line in Figure A2(b), can be achieved only with tape present on the illuminated face of the PG device. Thus, we conclude that the tape profoundly affects the capacitor-type behavior of the PG device.

In the time span of about 50 hours following the start of the activation of the PG device with conductive heat transfer from a $100 \Omega$ resistor, the voltage difference $\Delta V(t)$, temperature difference $\Delta T(t)$, and temperatures $T_{\text {hot }}(t)$ and $T_{\text {cold }}(t)$ of the activated and non-activated faces, respectively, are shown in Figures A3(a)-(d). We collected the particular set of data chosen using the PG device described above, finished with pink-colored gaffer tape, and set-up in the away architecture. Nevertheless, Figure A3 is representative of all the data collected in the away and toward architectures, with tape of all the available colors, and without tape. In all these cases, we observed $\Delta V(t), \Delta T(t), T_{\text {hot }}(t)$, and $T_{\text {cold }}(t)$ to be featureless and follow the same trends. The trend of $\Delta V(t)$ is symmetric to that of $\Delta T(t), T_{\text {hot }}(t)$, and $T_{\text {cold }}(t)$. This behavior differs from that observed for the PG device activated by the IR radiation and captured in Figure 5. In this case, $\Delta V(t)$ and the temperatures $T_{I R}(t)$ and $T_{\text {no-IR }}(t)$ of the illuminated and non-illuminated faces are strongly correlated, while $\Delta T(t)=T_{I R}(t)-T_{\text {no-IR }}(t)$ is flat and featureless.

The results in Figures A1-A3, together with those of Figures 3-5, further support that the electric and the entropic contribution to energy transfer from low power IR radiation to the PG device are decoupled.

\section{Appendix-2}

Figure 6 shows the hyperbolic instability revealed in the voltage difference $\Delta V(t)$ in the time span of about 30 hours following the start of the illumination of the PG device with IR radiation in the away architecture. We identify such hyperbolic instability when no correlation is found between $\Delta V(t)$ and the temperatures $T_{I R}(t)$ and $T_{n o-I R}(t)$ of the illuminated and non-illuminated faces of the PG device, respectively, and when $\Delta T(t)$ is flat. To demonstrate that hyperbolic instabilities are common in the interaction between IR radiation and a PG device, we collected a set of measurements with the PG device described in Appendix-1. With the AlO plate or

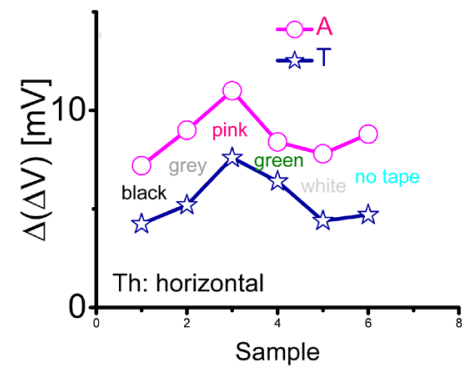

(a)

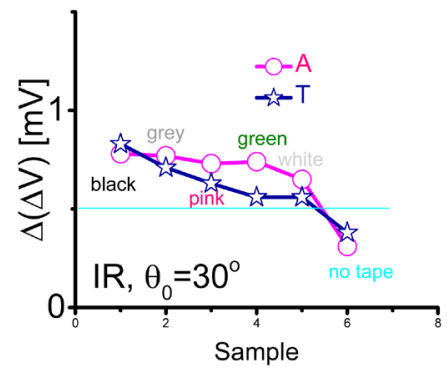

(b)

Figure A2. Jump in voltage $(\Delta(\Delta V))$ obtained performing measurements in the away $(A)$ and toward $(T)$ architectures on a PG device, described in Appendix-1, finished with an AlO plate covered with colored gaffer tape. The results compare data obtained in the 400 seconds immediately following the start the activation of the PG device with (a) conductive heat transfer from a $100 \Omega$ resistor or (b)IR radiation. The PG device was horizontally fixed on the sample holders in all the measurements. When the IR radiation was used, the PG device was positioned at an angle of incidence $\theta_{0}=30^{\circ}$ with respect to the IR radiation. The horizontal line at $0.5 \mathrm{mV}$ represents the minimum value of $\Delta(\Delta V)$ that can be obtained only by placing tape on the illuminated face of the PG device. 

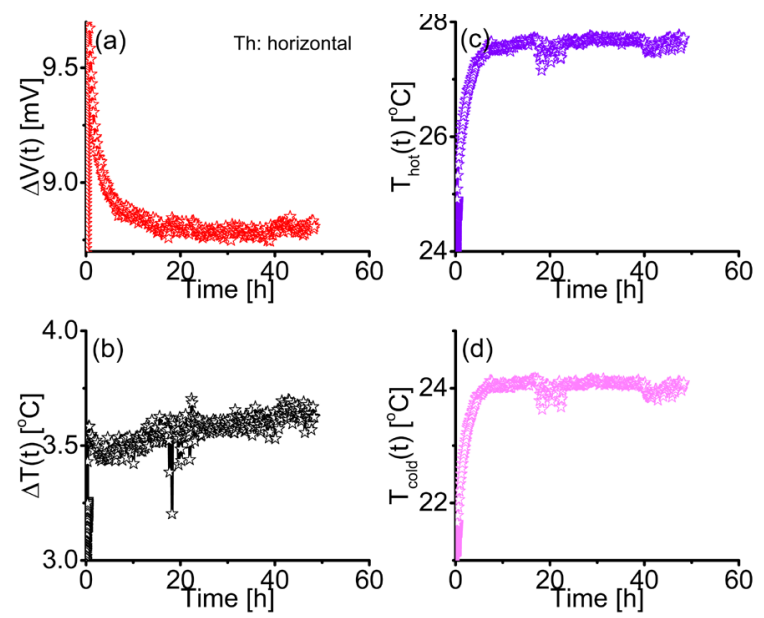

Figure A3. Panels (a), (b), (c), and (d) correspond to data collected from the away architecture in the whole time span of about 50 hours following the start of the activation of the PG device with conductive heat transfer from a $100 \Omega$ resistor. (a) Voltage difference $\Delta V(t)$. (b) Temperature difference $\Delta T(t)$. Temperatures $T_{\text {hot }}(t)$ (c) and $T_{\text {cold }}(t)$ (d) of the activated and non-activated faces, respectively, of the PG device. The PG device was horizontally fixed on the sample holders in these measurements and was finished with a layer of pink-colored gaffer tape on the AlO plate. The unit for time is the hour ([h]).

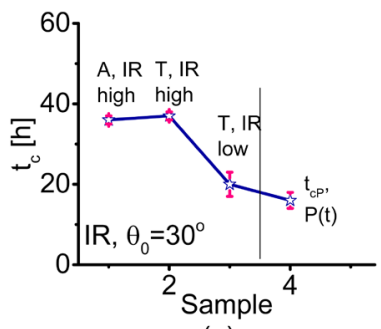

(a)

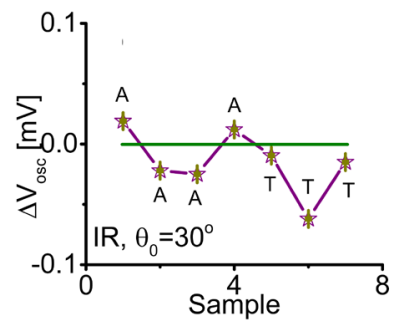

(b)

Figure A4. (a) Averages of the critical time ( $\left.t_{c}\right)$ and (b) amplitude ( $\left.\Delta V_{\text {osc }}\right)$ of the hyperbolic instability detected in the time span of about 50 hours following the start of the illumination of the PG device with IR radiation. The data were collected performing measurements on a PG device finished with an AlO plate, described in Appendix- 1 , and in the away (A) and the toward $(T)$ architectures. The PG device was positioned horizontally and at an angle of incidence $\theta_{0}=30^{\circ}$ with respect to the IR radiation. The average of the higher and lower values of $t_{c}$ found in the away and toward architectures are compared with the average value of $t_{c P}$ at $16 \pm 2 \mathrm{~h}$ for the power $P(t)$. In (b), the horizontal line separates the positive and negative $\Delta V_{\text {osc }}$.

the layer of tape facing the IR radiation, the surface density $\sigma(\mathbf{z}, t)$ refers to the charges in the AlO plate or in the tape. The results, summarized in Figure A4, report the average values of the critical time $\left(t_{c}\right)$ and amplitudes ( $\Delta V_{\text {osc }}$ ) of the revealed hyperbolic instabilities. We found a correlation neither between the values of $t_{c}$ in Figure A4(a) and the signs of $\Delta V_{\text {osc }}$ in Figure A4(b), nor between these quantities and the away or toward architectures. However, by observing Figure A4(a), we found that $t_{c}$ is typically located around $36 \pm 1 \mathrm{~h}$, which is about twice the magnitude of $t_{c P}$ at $16 \pm 2 \mathrm{~h}$ for the sinusoidal instability in $P(t)$ of the IR source reported in Table 2. In addition, we noted lower values for $t_{c}$ in Figure A4(a) located around $20 \pm 3 \mathrm{~h}$, i.e. in the neighborhood of $t_{c P}$. These observations highlight the correlations existing between the instabilities in $P(t)$ and $\Delta V(t)$ as already discussed in Section 3(e). Furthermore, in Figure A4(b) we noted randomness in the sign of $\Delta V_{\text {osc }}$, which we attribute to the loss of correlation between the phase of $P(t)$ and $\sigma(\mathbf{z}, t)$, and then between $\sigma(z, t)$ and $\Delta V(t)$. We predicted the loss of phase relationship in Section 3(b).

We summarize the findings in Figure $\mathbf{A} 4$ as follows: the hyperbolic instability in $\Delta V(t)$ is a common phenomenon, which is linked with the sinusoidal instability in the power $P(t)$ of the IR radiation. 